\title{
Pulmonary Artery Dissection Following Severe Pulmonary Artery Hypertension
}

\author{
Andro Diasmada*, Hendry Purnasidha Bagaswoto, Dyah Wulan Anggrahini \\ Department of Cardiology and Vascular Medicine, Faculty of Medicine, Universitas Gadjah Mada - \\ Dr. Sardjito Hospital, Yogyakarta, Indonesia \\ *correspondence: a.diasmada@gmail.com
}

\begin{abstract}
Background: Arterial dissection is caused by in-vessel hemorrhages, either secondary to intimal ruptures, to laceration or other pathology of the vasa vasorum. The dissection leads to the formation of a false dissecting lumen. Physio - pathologically it can lead to vessel occlusion with distal ischemia, artery - to - artery thromboembolism or hemorrhage. Pulmonary artery dissection is rare and has been reported in less than 100 patients. Most of them had been diagnosed post mortem, indicating that these patients suffer from cardiogenic shock or sudden death.

Aims: This case reported the event of pulmonary artery dissection in patients with severe pulmonal arterial hypertension NYHA III.

Case: Female, 36 y.o, felt shortness of breath and hemoptoe. One day before admission she felt shortness of breath, dyspnoe on effort, orthopnoe, and paroxysmal nocturnal dyspnea. She didn't feel chest pain, cough, and bloody sputum. One week before admission she felt dyspnea, cough with bloody sputum. She then went to RSI Klaten and was diagnosed as pulmonary arterial hypertension NYHA IV. She then referred to the cardiac clinic at Sardjito Hospital. Her past history she often cough with bloody sputum when she was child. She has been checked to lung clinic, sputum analysis had been obtained and the result was negative for tuberculosis. Blood pressure was $100 / 70$, pulse was $110 \mathrm{bpm}$, oxygen saturation was $97 \%$ on nasal canule oxygen $3 \mathrm{lpm}$.

Discussion: Dissection of the pulmonary artery is a rare condition. The most common cause is congenital heart disease (most commonly persistent ductus arteriosus) but other causes include primary or secondary pulmonary hypertension, vascular inflammatory disease, and catheter induced vessel wall injury. The reports of surviving patients demonstrate the feasibility of both conservative medical management (seven cases) and surgical repair (seven cases). Of the known case, 24 were diagnosed alive. Successfull repair of the pulmonary artery has been described in only seven cases. No clear consensus regarding how these patients are best best managed exist.

Conclusion: We conclude that managing patients with pulmonary arterial aneurysm/dissection medically in the acute phase may be appropriate. At the later stage, bilateral lung transplantation using the "en loc" technique, where the main pulmonary artery is preserved from the donor, can be performed.
\end{abstract}

Keywords: Pulmonary Arterial Hypertension; Pulmonary Artery Dissection 